\title{
Development of the Prototype of High Power Sub-THz Gyrotron for Advanced Fusion Power Plant (DEMO)
}

\author{
M.Morozkin $^{1}$, G.Denisov ${ }^{1}$, E.Tai ${ }^{2}$, E.Soluyanova ${ }^{2}$, A.Sedov ${ }^{1}$, A.Fokin ${ }^{1}$, A.Kuftin ${ }^{1}$, \\ A.Tsvetkov $^{1}$, M.Bakulin ${ }^{2}$, E.Sokolov ${ }^{2}$, V.Malygin ${ }^{1}$, M.Proyavin ${ }^{1}$, V.Zapevalov ${ }^{1}$, \\ O. Mocheneva, and M.Glyavin ${ }^{1}$ \\ ${ }^{1}$ Institute of applied physics RAS, Nizhny Novgorod, Russia, morozkin@ipfran.ru \\ ${ }^{2}$ Gycom Ltd., Nizhny Novgorod, Russia,
}

Next stage of the development of controlled nuclear fusion systems after the ITER project in accordance with the European Fusion Roadmap [1] is a demonstrational reactor DEMO, which should be the first thermonuclear reactor generating electrical energy. The parameters of the gyrotrons in the electron cyclotron heating system of the plasma of this reactor are not yet definitively specified, but frequencies of about $230-250 \mathrm{GHz}$ and power up to $2 \mathrm{MW}$ are discussed with an efficiency of more than $60 \%$ (full efficiency with energy recovery).

At present, most of the participants of the ITER project, which are involved in creating electroncyclotron plasma heating systems, have already begun developing prototypes of radiation sources that meet the requirements of the DEMO project [2]. In the Institute of Applied Physics and Gycom Ltd. for these purposes, a prototype gyrotron with a frequency of $250 \mathrm{GHz}$ and a radiation power of up to $200 \mathrm{~kW}$ in continuous mode was designed and tested (see fig. 1), intended for use in advanced fusion installations.

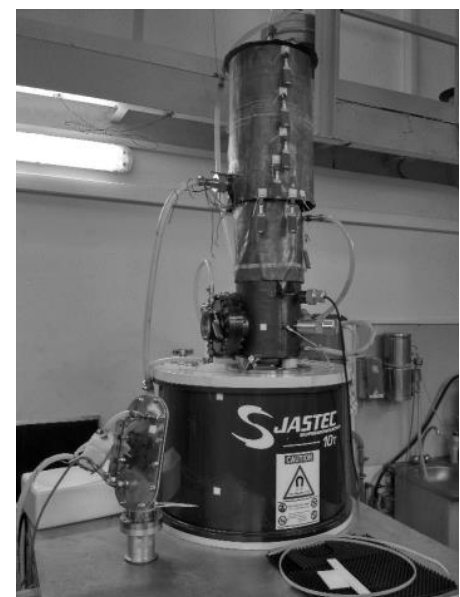

Fig. 1. Photo of the gyrotron in the cryomagnet.

The main factor limiting the output power in gyrotrons for plasma heating is the limitation of thermal loads on the cavity. Therefore, to increase the output power, it is necessary to increase the dimensions of the interaction space and use high-order modes. However, for the prototype gyrotron with an operating frequency of $250 \mathrm{GHz}$, the maximum size of the resonator and the electron-optical system was limited by the magnetic system. To generate the cyclotron frequency at the first harmonic, a magnetic field of more than $9.5 \mathrm{~T}$ is required, which is available in the cryomagnet at the IAP RAS, which has a warm bore of $100 \mathrm{~mm}$ in diameter. The warm bore diameter determined the dimensions of the electronic optics and cavity taking into account the technical requirements for cooling systems.

As a result, to ensure stable generation at the selected frequency, the $\mathrm{TE}_{19.8}$ mode was selected as the working mode with a cavity radius of $9.34 \mathrm{~mm}$.

Investigation of the coupling factors of the electron beam with the modes of the cylindrical cavity made it possible to determine the optimum radius of the electron beam in the cavity, which simultaneously provides both a large coupling coefficient with the working mode and the smallest coupling with parasitic modes. Due to the close coupling factors of the working mode $\mathrm{TE}_{19.8}$ and the parasitic modes $\mathrm{TE}_{18.8}$ and $\mathrm{TE}_{20.8}$, the optimal radius of the electron beam is $R_{\text {beam }}=3.93 \mathrm{~mm}$, which differs slightly from the radius of maximum coupling coefficient with the working mode $R_{\text {opt }}=3.85 \mathrm{~mm}$.

To ensure the effective interaction of the electron beam with the high-frequency field, the cavity length was optimized. The choice of length is determined by the compromise between the increase in the efficiency of the interaction of electrons with the RF field and the limitation of ohmic losses in the walls of the cavity (see Fig. 2). As a result of modeling, the length of the homogeneous section was chosen to be $\mathrm{L}=10$ $\mathrm{mm}$.
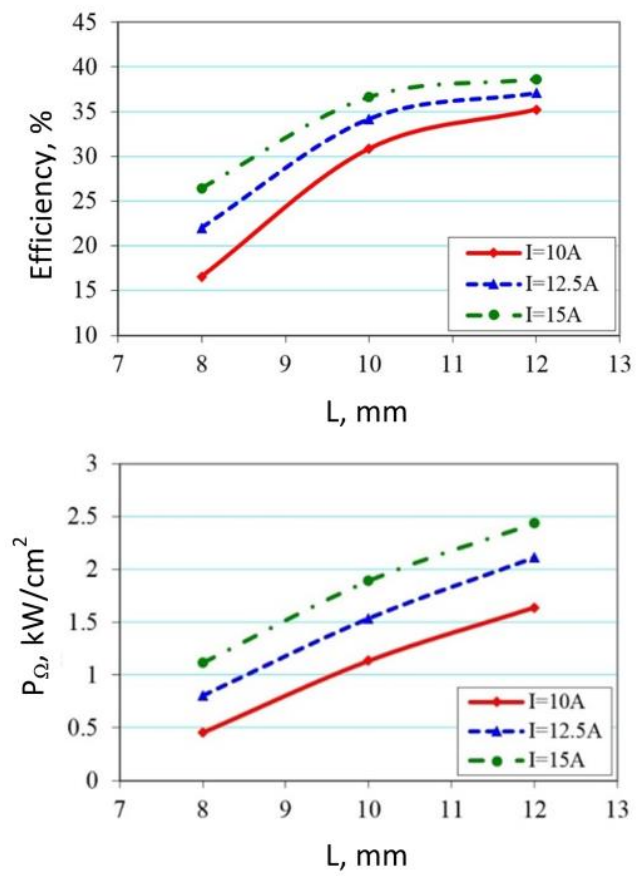

Fig. 2. Dependence of the efficiency and the density of ohmic losses on the length of a homogeneous part of the cavity 
The calculated parameters of the developed gyrotron are given in the following table.

Main parameters of the gyrotron

\begin{tabular}{|c|c|}
\hline Operating frequency, $\mathrm{GHz}$ & 250 \\
\hline Operating mode & $\mathrm{TE}_{19,8}$ \\
\hline Cyclotron harmonic & 1 \\
\hline Accelerating voltage $\mathrm{U}_{0}$ & $55 \mathrm{kV}$ \\
\hline Depressed collector potential & $30 \mathrm{kV}$ \\
\hline Magnetic field in the cavity & $9.6-9.7 \mathrm{~T}$ \\
\hline Beam current (nominal in CW mode) & $12 \mathrm{~A}$ \\
\hline Beam current (pulsed mode) & $20 \mathrm{~A}$ \\
\hline Cavity radius & $9.34 \mathrm{~mm}$ \\
\hline Cavity length (homogeneous part) & $10 \mathrm{~mm}$ \\
\hline Beam radius inside cavity & $3.93 \mathrm{~mm}$ \\
\hline Pitch factor, not less than & 1.1 \\
\hline
\end{tabular}

The tube is equipped with an internal quasioptical wave beam converter which provides a transformation of the operating mode into a Gaussian $\mathrm{TEM}_{00}$ beam and couples it radially to the vacuum window. The converter consists of a shaped waveguide, a quadratic mirror, four flat mirrors, and a synthesized mirror, which allows directing the wave beam in the output window.

The first experimental tests of the developed gyrotron were carried out at the IAP RAS on a highfrequency gyrotron setup equipped with a dry cryomagnet Jastec JMTD10T100 with a warm bore of $100 \mathrm{~mm}$ in diameter and a magnetic field of up to 10 $\mathrm{T}$. Due to the limitations of the power sources existing in the IAP RAS, the first experiments were carried out in a pulsed mode. For this tests a removable BN output window with a diameter of $66 \mathrm{~mm}$ was manufactured and installed. The thickness of the window was chosen to be about $3.1 \mathrm{~mm}$ in order to minimize the reflections at the operating frequency of $250 \mathrm{GHz}$. The pulse duration in the experiment was 20-40 $\mu \mathrm{s}$ with a repetition frequency of $10 \mathrm{~Hz}$. The power was measured with a water calorimetric dummy load equipped with thermal sensors in the inlet and outlet nozzles, the calibration was carried out with a heater in the circuit of a calorimeter with a known power of $100 \mathrm{~W}$. The output power of the gyrotron was calculated on the basis of the measurement of the steadystate average power in the pulsed mode, taking into account the duty cycle of 2500 .

The frequency of the output radiation was measured using a resonant-cavity wave meter. The registered value of $249.74 \mathrm{GHz}$ has been obtained at the following operating parameters: accelerating (cathode) voltage $\mathrm{U}_{0}=55 \mathrm{kV}$, beam current $\mathrm{I}_{\mathrm{b}}=12.5 \mathrm{~A}$, and magnetic field $\mathrm{B}=9.625 \mathrm{~T}$. These results are in agreement with the simulation data.

Reduction of the thermal load on the resonator during operation in the pulsed mode made it possible to test gyrotron with parameters exceeding the nominal design values. Thus, with an increase in the electron beam current, it was possible to reach a power of $330 \mathrm{~kW}$ at a beam current of $20 \mathrm{~A}$ and an accelerating voltage of $55 \mathrm{kV}$. The obtained experimental results are shown in Figures 3 and 4.

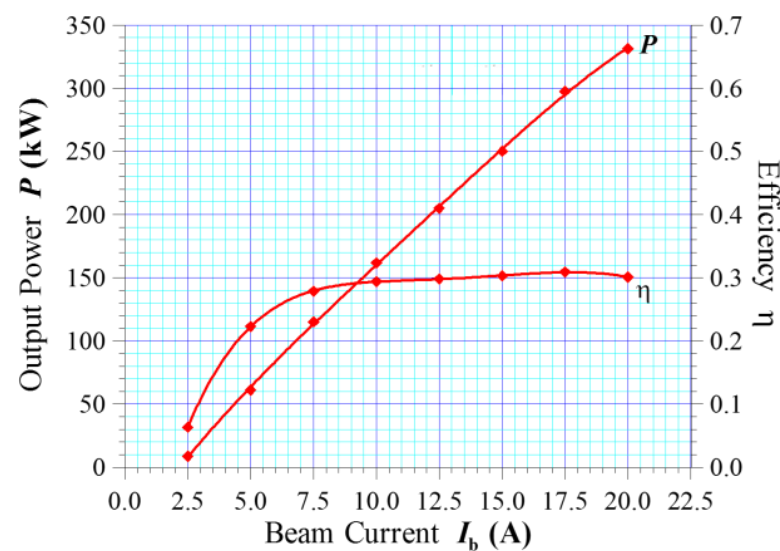

Fig. 3. Dependence of the output power and efficiency on the beam current, $\mathrm{U}_{0}=55 \mathrm{kV}$.

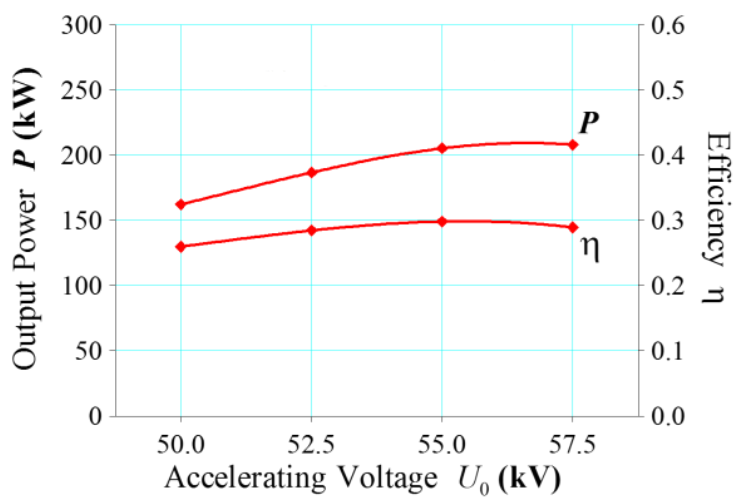

Fig. 4. Dependence of the output power and efficiency on the accelerating voltage, $\mathrm{I}_{\mathrm{b}}=12.5 \mathrm{~A}$.

Measurements of the transverse distribution of the microwave beam formed by the built-in quasi-optical gyrotron converter were made, and the content of the $\mathrm{TEM}_{00}$ wave (Gaussian beam) was estimated. The measurement was performed according to the thermal imaging technique [3] in several cross sections during the propagation of a wave beam. As the analysis showed, the content of a Gaussian wave beam in the reconstructed wave beam is $98.6 \%$.

Thus, the tested 250-GHz gyrotron showed output parameters close to the calculated ones: the power of $220 \mathrm{~kW}$ at a beam current of $12.5 \mathrm{~A}$ (corresponds to the beam current in a continuous mode) and $330 \mathrm{~kW}$ at a beam current of $20 \mathrm{~A}$. The generation efficiency was about 30\% (without energy recovery), the content of the $\mathrm{TEM}_{00}$ wave in the output beam is $98.6 \%$.

This work was supported by Russian scientific fund, grant 14-12-00887.

\section{References}

1. https://www.euro-fusion.org/eurofusion/roadmap/

2. J. Jelonnek et al. Design considerations for future DEMO gyrotrons: A review on related gyrotron activities within EUROfusion // Fusion Eng. Des. 2017. V. 123, P. 241-246.

3. S. O. Kuznezov, V. I. Malygin Determination of gyrotron wave beam parameters // Int. J. Infrared Millimeter Waves 1991. V. 12, No. 11, P. 1241-1252. 